\title{
Is Selective Mediolateral Episiotomy Protective Against Spontaneous Perineal Trauma and OASIS in Malaysian Tertiary hospitals? A Cross Sectional Study from the National Obstetrics Registry.
}

Shamala Devi Karalasingam ( $\sim$ shamaladevi@crc.gov.my )

National Obstetrics Registry, Institute Clinical Research, National Institute Health, Minstry of Health, Malaysia https://orcid.org/0000-0002-4992-906X

Ravichandran Jeganathan

Hospital Sultanah Aminah, Johor, Ministry of Health Malaysia

Ravindran Jegasothy

MAHSA University, Malaysia

Sharul Aiman Soelar

Sultanah Bahiyah Hospital, Malaysia

Daniel Reidpath

SEACO, Monash University Malaysia

Research article

Keywords: Perineal trauma, Selective Mediolateral Episiotomy, OASIS, Malaysian Tertiary hospitals

Posted Date: June 17th, 2020

DOI: https://doi.org/10.21203/rs.3.rs-32207/v1

License: (c) (i) This work is licensed under a Creative Commons Attribution 4.0 International License.

Read Full License 


\section{Abstract}

Introduction: Perineal trauma has short- and long-term debilitating effects on women. This study aimed to determine if selective episiotomy is protective against spontaneous perineal trauma and Obstetric anal sphincter injury following singleton vaginal birth in Malaysian tertiary hospitals and to identify the contributing factors.

Methods: This study used data from the Malaysian National Obstetrics Registry (NOR)which collects data from 11 tertiary hospitals in Peninsular Malaysia, 1 hospital in the Federal Territory Kuala Lumpur and in 2 from East Malaysia from 2010 to 2017. A total of 1,109,856 deliveries were analyzed of which 731,475 singleton vaginal deliveries with complete variables were included in this study. The primary objective were to identify contributing factors to spontaneous perineal trauma and Obstetric anal splinter injury (OASIS) and if selective episiotomy was effective in reducing perineal trauma.

Results: Prevalence of spontaneous perineal trauma in primiparous women was $23 \%$ while in multiparous women was $49.7 \%$. First and second-degree perineal tears were the commonest perineal injury followed by vaginal wall tears and labial tears. Less than $0.5 \%$ of the subjects had OASIS. Average episiotomy rate was $33.3 \%$. In both primiparous $(\mathrm{OR}=0.06 ; 95 \% \mathrm{Cl} ; 0.06)$ and multiparous women (OR $0.08 ; 95 \% \mathrm{Cl}: 0.03)$, selective episiotomy significantly reduces the risk spontaneous perineal trauma. The risk of OASIS, is reduced by episiotomy in primiparous women (OR 0.06; $95 \% \mathrm{Cl}$ : $0.05-0.07$ ) and in multiparous women (OR 0.19; 95\% Cl: 0.14-0.26).

Conclusion: Women have a higher risk of spontaneous perineal trauma when the accoucheur is a medical officer with less than 6 months' experience. In primiparous women spontaneous vaginal delivery is a risk for vaginal trauma and selective episiotomy appears to reduce the risk. All women with instrumental delivery are at risk of OASIS and selective episiotomy appears to reduce risk.

\section{Introduction}

Vaginal births are often associated with trauma to the genital tract. In some women the trauma is associated with obstetric anal sphincter injuries (OASIS) which include 3rd and 4th degree perineal tears. Trauma to the vaginal tract affects a women's physical and psychological health.

In the United Kingdom (UK) about $85 \%$ of women sustain some form of perineal trauma and OASIS ranging from $0.5 \%-7.0 \%$ during childbirth [1] The prevalence of OASIS ranged widely across countries from $0.1 \%$ (reported in China, Cambodia, India) to $15.0 \%$ (reported in the Philippines) [2]. Forceps and Vacuum deliveries are a risk factor for OASIS. It occurs in $5-7 \%$ of spontaneous vaginal births and increases with operative vaginal delivery to $12-14 \%$ [3]

A survey done in 2011 in UK quoted the National rates of OASIS as $2.9 \%$ with incidence in primiparous women at $6.1 \%$ and lower in multiparous women at 1.7\% [4] Episiotomies affect the perineal body but lacerations can occur in the labia, vaginal wall, and anterior vaginal structures in addition to the perineal 
body. Minor tears may heal without any issues however those that involve muscle and sphincter will require surgical repair. Risk factors include induction of labour, parity, shoulder dystocia, a prolonged second stage, assisted delivery and macrosomia.

A Cochrane review concluded that for women where an unassisted vaginal birth was anticipated, a policy of selective episiotomy may result in $30 \%$ fewer women experiencing severe perineal/vaginal trauma (RR $0.70,95 \% \mathrm{Cl} 0.52$ to $0.94 ; 5375$ women; eight RCTs; low-certainty evidence) [5]. Episiotomies were performed to decrease the frequency of perineal tears but there is also evidence that it causes pelvic floor dysfunction. The World Health Organization does not recommend routine but selective episiotomy and an acceptable rate is difficult to determine [6] Restrictive use of episiotomy in uncomplicated vaginal births, compared with routine episiotomy, is associated with reduced risk of posterior perineal trauma [7] based on the data obtained in the English randomized controlled trial published in 1984 [8]. This study looks at the prevalence of spontaneous perineal trauma and OASIS in Malaysian tertiary hospitals, and examined the evidence that selective episiotomy reduced the rate of perineal trauma.

\section{Methods}

This is a retrospective study based on the data from the Malaysian NOR, which is a clinical registry that compiles obstetric data on all births from 11 tertiary State hospitals in Peninsular Malaysia, one hospital in the Federal Territory Kuala Lumpur and in two State tertiary hospitals in East Malaysia. A complete description of NOR can be found in annual reports 2010[9] and 2011-2012 [10] and the website[http://www.acrm.org.my/nor/] Ethical approval for the NOR was provided by the medical research ethics committee of the Ministry of Health, Malaysia (Approval number NMRR 15-620-25530) This study was conducted in compliance with the ethical principles outlined in the Declaration of Helsinki and Malaysian Good Clinical Practice Guideline. No personal information of participants was disclosed.

All singleton vaginal deliveries (spontaneous vertex deliveries, assisted vaginal deliveries and vaginal breech deliveries) from the participating hospitals were included that occurred between January 1, 2010 and December 31, 2017. Caesarean sections (CS), all multiple pregnancies, and incomplete variables were excluded. Perineal trauma was categorized as follows: (1) 1st and 2nd degree tears, (2) 3rd and 4th degree tears, (3) cervical tears, (4) labial tears, (5) clitoral tears (6) vaginal wall tears and (7) episiotomy. The primary objective was to look at the prevalence of spontaneous perineal trauma in Malaysian tertiary hospitals. The secondary objectives were to identify possible contributing factors to spontaneous perineal trauma, identify risk factors for OASIS and gain insights into the potential effectiveness of selective episiotomy in reducing perineal trauma.

The analyses were performed with $\mathrm{R}$ version 3.5.2. [11. Descriptive statistics were obtained. The prevalence of episiotomy in women with a singleton vaginal delivery from year 2010 to 2017 was tested using trend analysis for proportions. Simple logistic regression analysis was used to explore the crude odds ratio of spontaneous perineal trauma and OASIS in women with a singleton vaginal delivery. The odds ratio of spontaneous perineal trauma and OASIS was adjusted in subsequent multivariable analysis 
for parity, age group, birthweight, Asian body mass index classifications [12], level of accoucheur, assisted vaginal delivery, spontaneous vertex delivery, breech delivery, episiotomy, shoulder dystocia and prolonged 2 nd stage. A probability value of less than 0.05 ( $p$-value $<0.05)$ was considered statistically significant [13].

\section{Results}

From 2010 to 2017 there were a total of 1,109,856 deliveries from the participating hospitals. A total $292,805(26.4 \%)$ CS, 23,080 (2.1\%) multiple pregnancies and 62,496 (5.6\%) deliveries with incomplete variables $(7.8 \%)$ were omitted from the study. Of the 731,475women included in the study, $242,098(33.1 \%)$ were primiparous and $489,377(66.9 \%)$ were multiparous. The mean age of women in this study was 28.5 years. Table 1 shows descriptive analysis. The prevalence of spontaneous perineal trauma in primiparous women was $23 \%$ and $49.7 \%$ in multiparous women. First and second-degree perineal tears were the most common perineal injury (34\%) followed by vaginal wall tears $(4.5 \%)$ and labial tears (4.2\%). Less than $0.5 \%$ of the subjects had OASIS and the average episiotomy rate was $33.3 \%$.

Table 2 shows the association of potential risk factors and spontaneous perineal trauma in primiparous women. The crude odds ratios show that every level of accoucheur was associated with higher odds of spontaneous perineal trauma compared to a specialist. The association, however, did not hold in an adjusted model. The adjusted odds ratio shows that after controlling for other factors only Medical Officers with 6 months or less obstetrics experience had significantly greater odds of spontaneous perineal trauma than a specialist, $\mathrm{OR}=2.09 ; 95 \% \mathrm{Cl}: 1.91-2.09$. Nurses had a significantly lower odds of a delivery associated with spontaneous perineal trauma than a medical specialist (Staff nurse OR $=0.80$; 95\% Cl:0.73-0.88; Community nurse OR $=0.90 ; 95 \% \mathrm{Cl}: 0.82-0.99)$. BMI was not significantly associated with spontaneous perineal trauma.

Table 3 shows the association of potential risk factors and spontaneous perineal trauma in multiparous women. There was a greater risk of spontaneous perineal trauma in multiparous women associated with a Medical officer who had Obstetric experience of 6 or fewer months ( $\mathrm{OR}=1.81 ; 95 \% \mathrm{Cl}: 1.69-1.94)$ compared to a specialist. In both primiparous $(\mathrm{OR}=0.06 ; 95 \% \mathrm{Cl} ; 0.06)$ and multiparous women (OR 0.08; $95 \% \mathrm{Cl}: 0.08)$, episiotomy reduces the risk of spontaneous perineal trauma.

Table 4 shows the odds ratio for the association of OASIS in primiparous women. A higher risk was seen in women aged $21-40$ years (OR 2.39; $95 \% \mathrm{Cl}$ : 1.93-3.00). There was no increase risk in relation to level of accoucheur but there was an increased risk with vacuum assisted delivery (OR 15.81: 95\% Cl:6.8338.43 ) and Forceps assisted delivery (OR $29.87 ; 95 \% \mathrm{Cl}: 12.74-71.92)$. Shoulder dystocia increased the risk of OASIS (OR 8.12; 95\% Cl: 4.50-13.78) as did a birthweight of $4000 \mathrm{~g}$ and more (OR $4.48 ; 95 \% \mathrm{Cl}$ : $2.89-6.67)$.

Table 5 shows the odds ratio for the association of OASIS in multiparous women. There was a higher risk of OASIS following vacuum assisted delivery (OR 11.71; 95\% Cl: 3.92-41.11) and forceps assisted 
delivery (OR 22.73; 95\% Cl 7.01-77.42). Shoulder dystocia (OR 7.75; 95\% Cl: 4.99-11.66) and a birthweight of $4000 \mathrm{~g}$ and more (OR 4.38; $95 \% \mathrm{Cl}: 3.29-5.74$ ) also had a greater odd of OASIS. There was a dose response effect with increasing $\mathrm{BMI}$ and increased risk of OASIS in multiparous women. Episiotomy reduced the risk of OASIS in primiparous women (OR $0.06 ; 95 \% \mathrm{Cl}: 0.05-0.07$ ) and in multiparous women (OR 0.19; 95\% Cl: 0.14-0.26).

\section{Discussion}

This study looks at the prevalence and risk factors of spontaneous perineal trauma and OASIS in Malaysian tertiary hospitals and to consider whether selective episiotomy was protective against spontaneous perineal trauma and OASIS. There have been conflicting findings from previous studies. It has been reported in some studies that spontaneous perineal trauma was higher in primiparous births [14] while others have reported that multiparous births were associated with having a higher risk of perineal trauma. [15]. Studies [16] have shown mediolateral episiotomies to be associated with increased risk of OASIS. De Leeuw et al [17] shows mediolateral episiotomy reduces OASIS for instrumental delivery. Cochrane review [18] concluded that selective episiotomy reduces women experiencing severe perineal trauma and routine episiotomy reduces perineal trauma is not justified. In the Malaysian NOR data, in primiparous birth every 2 in 10 women had a spontaneous perineal trauma. The risk factors for spontaneous perineal trauma in primiparous births were associated with an inexperienced accoucheur, a medical officer with 6 months or less experience. A spontaneous vaginal delivery itself was also a risk for perineal trauma in this group. In multiparous women almost $50 \%$ of women had a spontaneous perineal trauma. The risk factors in this group were similar to those nulliparous women, however spontaneous vaginal delivery was not a risk for perineal trauma. The episiotomy rates in Malaysian tertiary hospitals from 2010 to 2017 dropped from 36-34.1\%. 7 out of 10 primiparous women received an episiotomy while only 1 out of 10 in multiparous women had an episiotomy. There has been a reluctance for change where episiotomy has been practiced routinely in primiparous women although there has been a change in recommendation against routine episiotomies as it causes worse tearing than might occur naturally during childbirth.[5] This study shows a slight change in practice. Larger angle of mediolateral episiotomy is said to be associated with a lower risk of third-degree tear to minimize the risk of sphincter disruption. [19] In Malaysia, only mediolateral episiotomies, 45区 away from the midline are done and this is evident from the Midwifery curriculum. Varying episiotomy rates may be reflective of the quality of care as well as variations in clinical practice in a country. Monitoring the rates as well as advocating selective as compared to routine episiotomy also reflects commitment to quality of care and adherence to best practices. There has been a change in recommendation against routine episiotomies as it causes worse tearing than might occur naturally during childbirth. [5]. Selective episiotomy had a protective effect in both primiparous and multiparous women. This study suggests that all women have a higher spontaneous perineal trauma when the accoucheur is a medical officer with less than 6 months' experience. In nulliparous women spontaneous vaginal delivery is a risk for vaginal trauma and selective episiotomy appears to reduce the risk. Reducing episiotomy rates should not be at the expense of increasing perineal trauma and OASIS. All women with instrumental delivery, shoulder dystocia and 
macrosomic baby should have an episiotomy done to reduce the incidence of OASIS. Junior Doctors must be supervised to reduce the incidence of spontaneous perineal trauma.

\section{Conclusion}

Women should be effectively screened and episiotomy advocated for the indication stated to reduce the incidence of perineal trauma.

\section{Limitation of study}

The Malaysian National Obstetrics Registry collects data from only 14 tertiary hospitals

\section{Abbrevations}

OASIS: Obstetrics anal sphincter injuries; UK: United Kingdom; RCT: Randomized control trial; NOR: National Obstetrics Registry; CS: Caesarean Section; BMI: Body Mass Index.

\section{Declarations}

\section{Acknowledgement}

The authors would like to thank the Director General of Health for his support for the Malaysian NOR. We would also like to thank all site coordinators and site assistants of NOR for their efforts to capture data.

\section{Funding}

Nil

\section{Availability of data and materials}

The legal data management requirements of the Ministry of health Malaysia do not allow data to be placed on an open access facility but we encourage collaborative research. The data is available on request for legitimate research. The data release form is available on the NOR website: http://www.acrm.org.my/nor/

\section{Authors contributions}

SDK and RJ oversaw the collection and curation of the NOR data. SDK, RJ and RJS conceived the idea for the paper and further developed by DDR. SAS conducted the analysis with input from SDK, RJ, RJS and DDR.SDK wrote the initial draft. All authors contributed to subsequent drafts and approved final version of the paper.

\section{Authors information}


Dr Shamala Devi Karalasingam is an Obstetrician and Gynaecologist and the Project Manager of the Malaysian National Obstetrics Registry. Dr Ravichandran Jeganathan is the Head of Obstetrics and Gynaecology at Sultanah Aminah Hospital and Principal Investigator of the Malaysian National Obstetrics Registry.

Dr Ravindran Jegasothy is an Obstetrician and Gynaecologist, currently the Dean, Faculty of Medicine, MAHSA University, Malaysia. Sharul Aiman Soealar currently a biostatistician in the Clinical Research Centre at Sultanah Bahiyah Hospital Kedah and Prof Daniel Reidpath is professor of population health and an epidemiologist.

\section{Competing interests}

The authors declare that they have no competing interest.

\section{Consent for publication}

The paper has approval for publication from the Director General of Health, Malaysia

\section{Ethics approval and consent to participate}

Ethical approval for the research was obtained by the Medical Research and Ethics committee of the Ministry of Health, Malaysia (Approval number NMRR15-620-25530).

Patient consent was deemed unnecessary for the collection and use of NOR data.

\section{References}

1. Kettle C, Tohill S: Perineal care. Clin Evid (Online). 2008, http://www.ncbi.nlm.nih.gov/pubmed/19445799 (accessed 20th June 2012) whilst 3rd and 4th degree perineal tear (OASIS).

2. Prevalence Mori A, Zhang R, Souza J, Gülmezoglu JP. and risk factors for third- and fourth-degree perineal lacerations during vaginal delivery: a multi-country study.Hirayama F1, Koyanagi. AM. BJOG. 2012 Feb;119(3):340-7.

3. A randomized control trial. evaluating the prevalence of Obstetrical anal sphincter injury in primi in routine versus selective mediolateral episiotomy. Amar Sulaiman et al. Saudi Med J 2013.

4. Annual report 2016, Swedish pregnancy registry.

5. Cochrane review. Selective versus routine use of episiotomy for vaginal birth, February 2017.

6. WHO recommendation. on episiotomy policy.17 February 2018.

7. Liljestrand J. Episiotomy for vaginal birth: RHL commentary (last revised: 20 October 2003). The WHO Reproductive Health Library; Geneva: World Health Organization.

8. Clin Res 
Br Med J (Clin Res editor.. 1984 Sep 8; 289(6445): 587-590.West Berkshire perineal management trial. J Sleep, Grant A, Garcia J, Elbourne D, Spencer J, Chalmers I.

9. Ravichandran J. SD Karalasingam Editors. National Obstetrics report 2010.

10. Ravichandran J. SD Karalasingam Editors. National Obstetrics report 2011-2012.

11. Team RC. R: A language and environment for statistical computing. Vienna: Austria:R Foundation for Statistical Computing; 2014.

12. Importance of Adopting BMI Classifications Using Public Health Action Points to Delineate Obstetric Risk Factors Resulting in Worsening Obstetric Outcomes Among Asian Population 68

173

178

10.1007/s13224-017-1000-9

Epub 2017 May 4.

Importance of Adopting BMI Classifications Using Public Health Action Points to Delineate Obstetric Risk Factors Resulting in Worsening Obstetric Outcomes Among Asian Population. Ganeshan M, Bujang MA, Soelar SA, Karalasingam SD, Suharjono H, Jeganathan R. J Obstet Gynaecol India. 2018 Jun;68(3):173-178. Doi: 10.1007/s13224-017-1000-9. Epub 2017 May 4.

13. How to Report Statistics in Medicine. Annotated Guidelines for Authors, Editors, and Reviewers2006. Thomas A. Lang, Michelle Secic.

14. Perineal Trauma in Primiparous Women with Spontaneous Vaginal Delivery. Episiotomy or SecondDegree Perineal Tear? Irene Mora-Hervás, Emília Sánchez,Francisco Carmona, Montserrat EspuñaPons. International Journal of Women's Health Reproduction Sciences Vol. April 2015;3(2):84-8.

15. Incidence of and. risk factors for perineal trauma: a prospective observational study. Lesley A Smith, Natalia Price, Vanessa Simonite and Ethel E Burns. BMC Pregnancy Childbirth. 2013.

16. Murphy DJ, Macleod M, BahI R, Goyder K. L.Howarth, BStrachan. A randomized controlled trial of routine versus restrictive use of episiotomy at operative vaginal delivery: a multicenter pilot study. Obstet Gynecol Survey. 2009;64(4):220-1.

17. De Leeuw J, De Wit C, Bruinse H, Kuijken J. Mediolateral episiotomy reduces the risk for anal sphinter injury during operative vaginal delivery. BJOG2008; 115:104-8.

18. Jiang H, Qian X, Carroli G, Garner P. Selective versus routine use of episiotomy for vaginal birth. Cochrane 2017.

19. Eogan M, Daly L, O'Connell PR. C O'Herlihy Does the angle of episiotomy affect the incidence of anal sphincter injury? BJOG 2006 Feb;113(2):190-4.

\section{Figures}




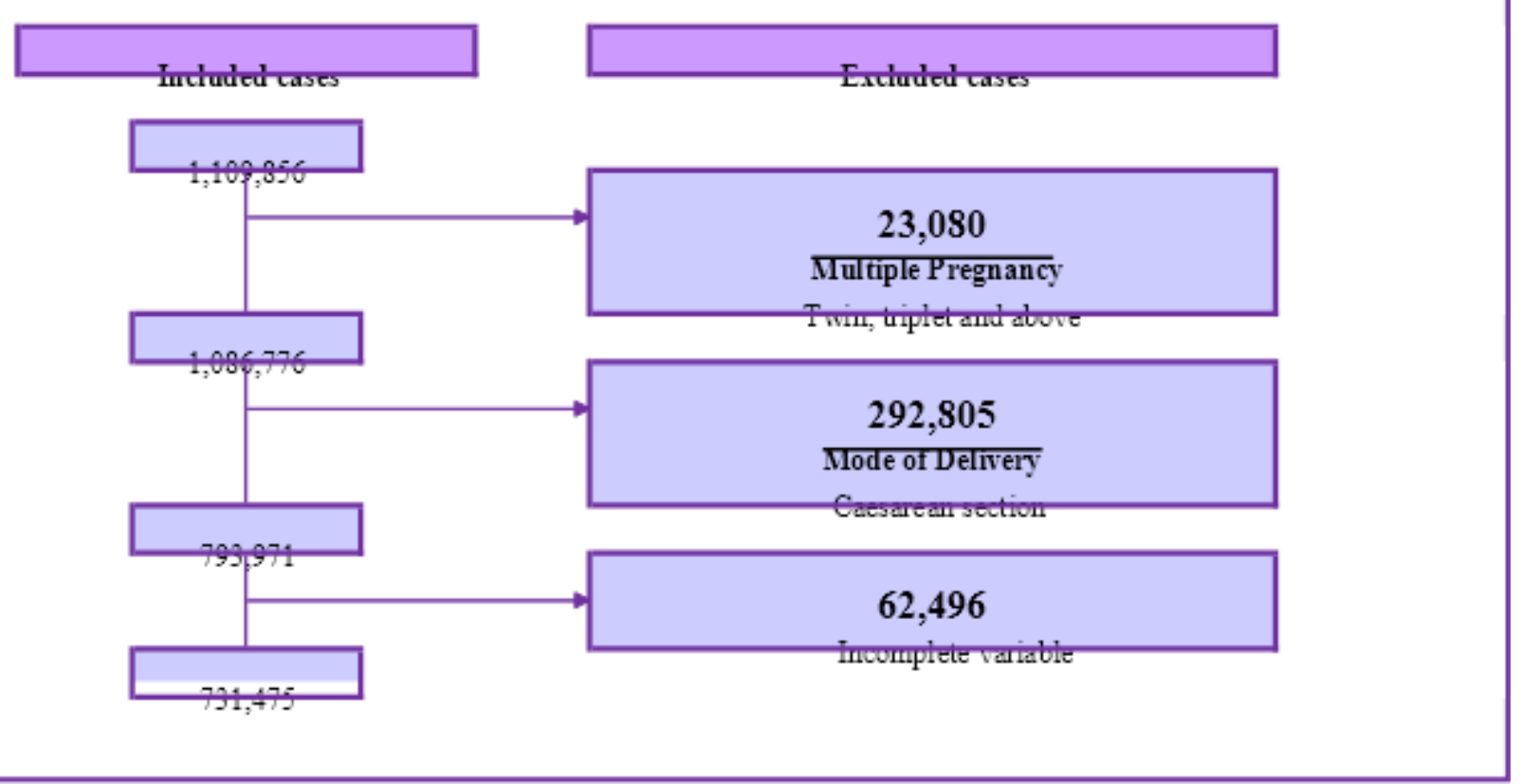

Figure 1

Flow chart of study selection process

\section{Episiotomy rates 2010-2017}

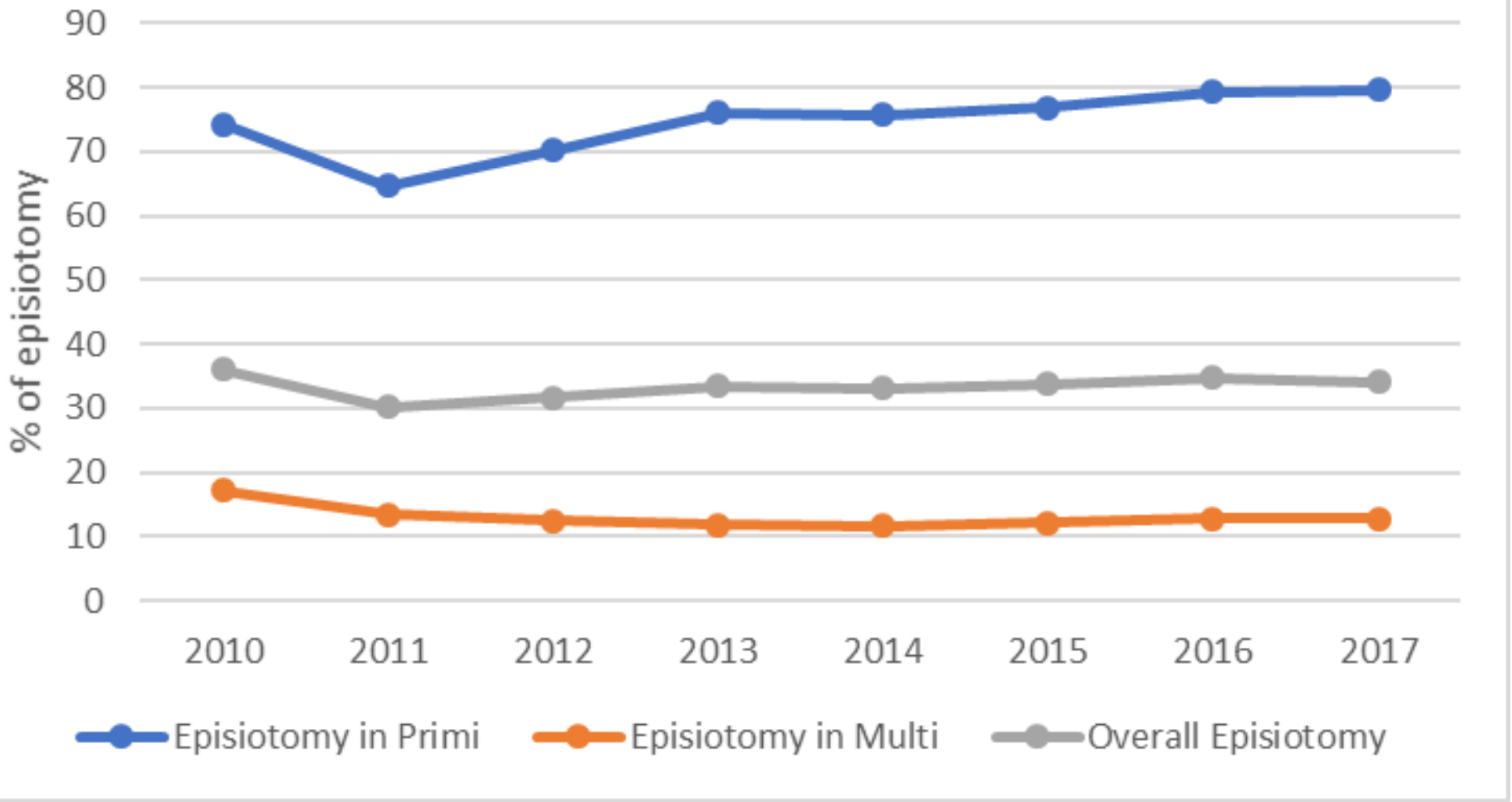

\section{Figure 2}

Prevalence of episiotomy in women with a singleton vaginal delivery by year and parity. 